\title{
The first experimental observation of odderon exchange
}

\author{
F. Nemes on behalf of the DO and TOTEM collaborations \\ CERN (Also at Wigner RCP, Hungary) \\ fnemes@cern.ch
}

We describe an analysis comparing the $\mathrm{p} \overline{\mathrm{p}}$ elastic cross section as measured by the D0 Collaboration at a center-of-mass energy of $1.96 \mathrm{TeV}$ to that in pp collisions as measured by the TOTEM Collaboration at 2.76, 7, 8, and $13 \mathrm{TeV}$ using a data-driven approach. The TOTEM cross sections, extrapolated to a center-of-mass energy of $\sqrt{s}=1.96 \mathrm{TeV}$, are compared with the D0 measurement in the region of the diffractive minimum and the second maximum of the pp cross section. The two data sets disagree at the $3.4 \sigma$ level and thus provide evidence for the t-channel exchange of a colorless, C-odd gluonic compound, also known as the odderon. We combine these results with a TOTEM analysis of the same C-odd exchange based on the total cross section and the ratio of the real to imaginary parts of the forward elastic strong interaction scattering amplitude in pp scattering for which the significance is between $3.4 \sigma$ and $4.6 \sigma$. The combined significance is larger than $5 \sigma$ and is interpreted as the first observation of the exchange of a colorless, C-odd gluonic compound.

LHCP 2021

7-12 June, 2021

Paris, France 


\section{COMPARISON OF ELASTIC PP TOTEM AND PP̄ DO DATA}

This proceedings summarizes the data-driven comparison of the pp elastic cross section extrapolated from the TOTEM measurements at the LHC to the $\mathrm{p} \overline{\mathrm{p}}$ cross section measured at the Tevatron by the D0 collaboration. A difference in these cross sections in the multi- $\mathrm{TeV}$ range would constitute a direct demonstration for the existence of a colourless $\mathrm{C}$-odd gluonic compound state, the odderon. More details can be found in Ref. [1]. The D0 Collaboration [2] measured the $\mathrm{p} \overline{\mathrm{p}}$ elastic differential cross section at $\sqrt{s}=1.96 \mathrm{TeV}$ [3]. The TOTEM Collaboration [4] at the CERN LHC measured the differential elastic pp cross sections at $\sqrt{s}=2.76$ [5], 7 [6], 8 [7] and 13 [8] TeV. Figure 1a shows the TOTEM differential cross sections used in this study as functions of $|t|$. All pp cross sections show a common pattern of a diffractive minimum ("dip") followed by a secondary maximum ("bump") in $\mathrm{d} \sigma / \mathrm{d} t$. Figure $1 \mathrm{~b}$ shows the ratio $\mathrm{R}$ of the differential cross sections measured at the bump and dip locations as a function of $\sqrt{s}$ for ISR [9, 10], Spp̄S [11, 12],

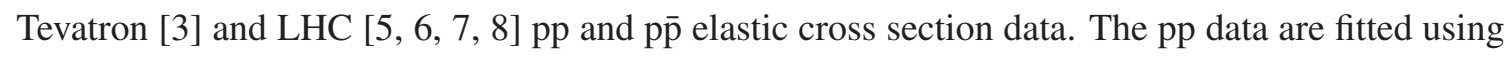
the formula $R=R_{0}+a_{0} \exp \left(b_{0} \sqrt{s}\right)$. There is no discernible dip or bump in the D0 p $\overline{\mathrm{p}}$ cross section and the estimated D0 $R=1.0 \pm 0.2$ value differs from the pp ratio by more than $3 \sigma$. The $\mathrm{R}$ values

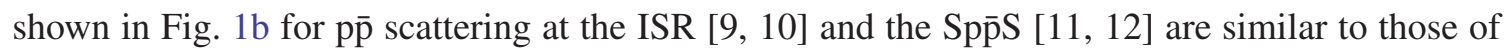
the D0 measurement.

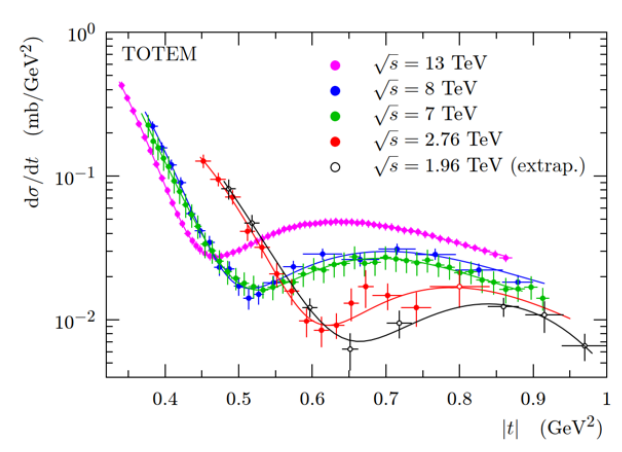

(a)

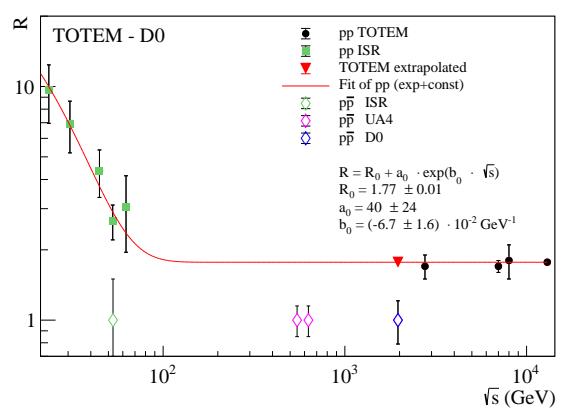

(b)

Figure 1: (a) The TOTEM measured pp elastic cross sections as functions of $|t|$ at 2.76, 7, 8, and $13 \mathrm{TeV}$ (full circles), and the extrapolation (discussed in the text) to $1.96 \mathrm{TeV}$ (empty circles). The lines show the double exponential fits to the data points (see text). (b) The ratio $\mathrm{R}$ of the cross sections at the bump and dip as a function of $\sqrt{s}$ for $\mathrm{pp}$ and $\mathrm{p} \overline{\mathrm{p}}$. The $\mathrm{pp}$ data are fitted to the function noted in the legend.

Motivated by the features of the pp elastic $\mathrm{d} \sigma / \mathrm{d} t$ measurements, we define a set of eight characteristic points as shown in Fig. 3a. For each characteristic point, we identify the values of $|t|$ and $\mathrm{d} \sigma / \mathrm{d} t$ at the closest measured points to the characteristic point, thus avoiding the use of model-dependent fits. If two neighboring measurements are nearly identical, we use their average. This leads to a distribution of $|t|$ and $\mathrm{d} \sigma / \mathrm{d} t$ values as a function of $\sqrt{s}$ for all characteristic points as shown in Fig. 2a and 2b. The uncertainties correspond to half the bin size in $|t|$ (comparable to the $|t|$ resolution) and to the published uncertainties on the cross sections.

The values of $|t|$ and $\mathrm{d} \sigma / \mathrm{d} t$ as functions of $\sqrt{s}$ for each characteristic point are fitted using the functional forms, so $|t|=a \log (\sqrt{s})+b$ and $(\mathrm{d} \sigma / \mathrm{d} t)=c \sqrt{s}+d$ respectively. The parameter values are determined for each characteristic point separately and the same functional form describes 


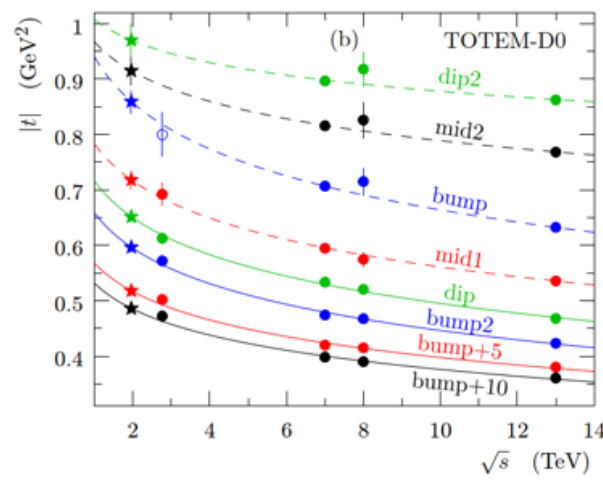

(a)

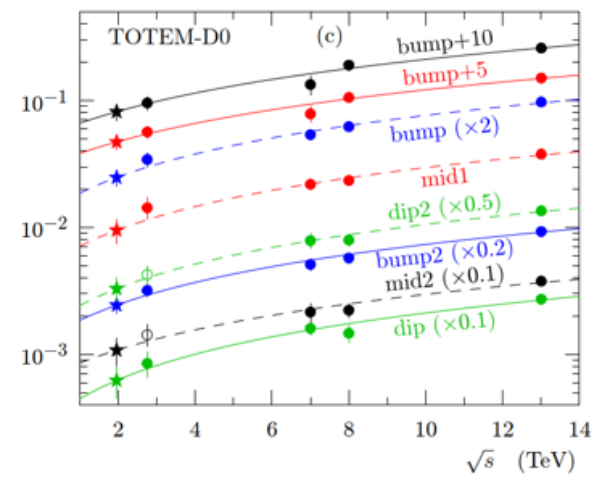

(b)

Figure 2: Characteristic points in (a) $|t|$ and (b) $\mathrm{d} \sigma / \mathrm{d} t$ from TOTEM measurements at 2.76, 7, 8, and $13 \mathrm{TeV}$ (circles) as a function of $\sqrt{s}$ extrapolated to Tevatron center-of-mass energy (stars). On (b), a multiplication factor indicated in parenthesis is applied in order to distinguish the different fits. Filled symbols are from measured points; open symbols are from extrapolations or definitions of the characteristic points.

the dependence for all characteristic points. The $\chi^{2}$ values for the majority of fits are close to 1 per degree of freedom. The $|t|$ and $\mathrm{d} \sigma / \mathrm{d} t$ values for the characteristic points for pp interactions extrapolated to $1.96 \mathrm{TeV}$ are displayed as stars in Fig. 2.

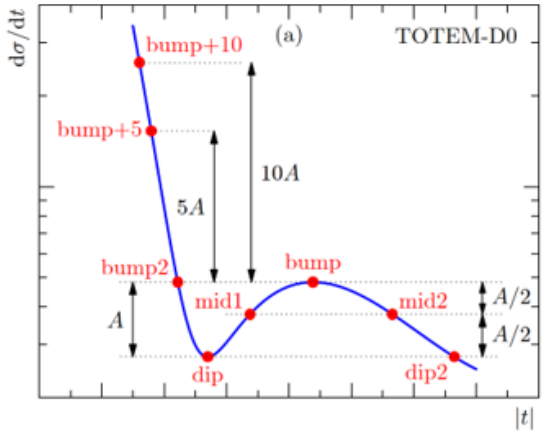

(a)

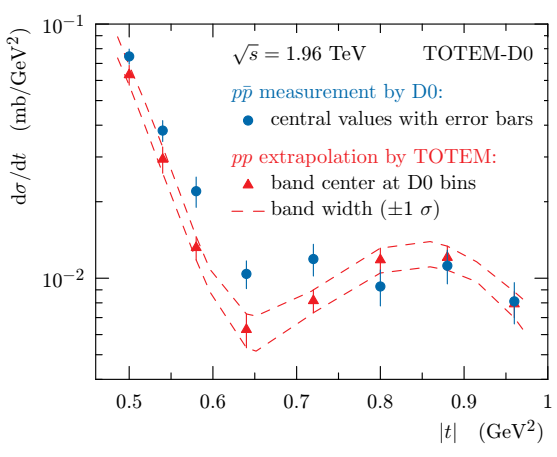

(b)

Figure 3: (a ) Schematic definition of the characteristic points in the TOTEM differential cross section data. The quantity $A$ represents the vertical distance between bump and dip. (b) Comparison between the D0 p $\bar{p}$ measurement at $1.96 \mathrm{TeV}$ and the extrapolated TOTEM pp cross section, rescaled to match the OP of the D0 measurement. The dashed lines show the $1 \sigma$ uncertainty band on the extrapolated pp cross section.

To compare the extrapolated pp elastic cross sections with the p $\bar{p}$ measurements, we fit the extrapolated pp cross section with the function

$$
h(t)=a_{1} e^{-a_{2}|t|^{2}-a_{3}|t|}+a_{4} e^{-a_{5}|t|^{3}-a_{6}|t|^{2}-a_{7}|t|}
$$

to allow interpolation to the $t$-values of the D0 measurements in the range $0.50 \leq|t| \leq 0.96 \mathrm{GeV}^{2}$. This function also provides good fit for the measured pp cross sections, see Fig. 1a. We evaluate the pp extrapolation uncertainty from Monte Carlo (MC) simulation in which the cross section 
values of the eight characteristic points are varied within their Gaussian uncertainties and new fits given by Eq. (1.1) are performed. We scale the pp extrapolated cross section so that the optical point $(\mathrm{OP}), \mathrm{d} \sigma / \mathrm{d} t(t=0)$, is the same as that for $\mathrm{p} \overline{\mathrm{p}}$. The TOTEM total cross sections are fitted and extrapolated to $1.96 \mathrm{TeV}$ using the function $\sigma_{\mathrm{tot}}=b_{1} \log ^{2}(\sqrt{s} / 1 \mathrm{TeV})+b_{2}$. The OP is calculated using the optical theorem. The final scale factor on the TOTEM extrapolated cross-section is $0.954 \pm 0.076$; assumption of equal OP for $p p$ and $p \bar{p}$ consistent within the experimental OP uncertainties. Systematic uncertainties arising from departures from the hypothesis of equal OPs are included [1]. We perform a $\chi^{2}$ test to examine the probability for the D0 and TOTEM differential elastic cross sections to agree using their covariance matrices, see Fig. 3b. Given the constraints on the normalization and logarithmic slopes, the $\chi^{2}$ test with six degrees of freedom yields the $p$-value of 0.00061 , corresponding to a significance of $3.4 \sigma$. We make a cross check of this result using an adaptation of the Kolmogorov-Smirnov test in which correlations in uncertainties are taken into

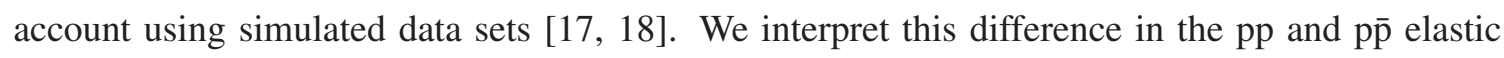
differential cross sections as evidence that two scattering amplitudes, even and odd under crossing, are present, identified as Pomeron and odderon exchanges.

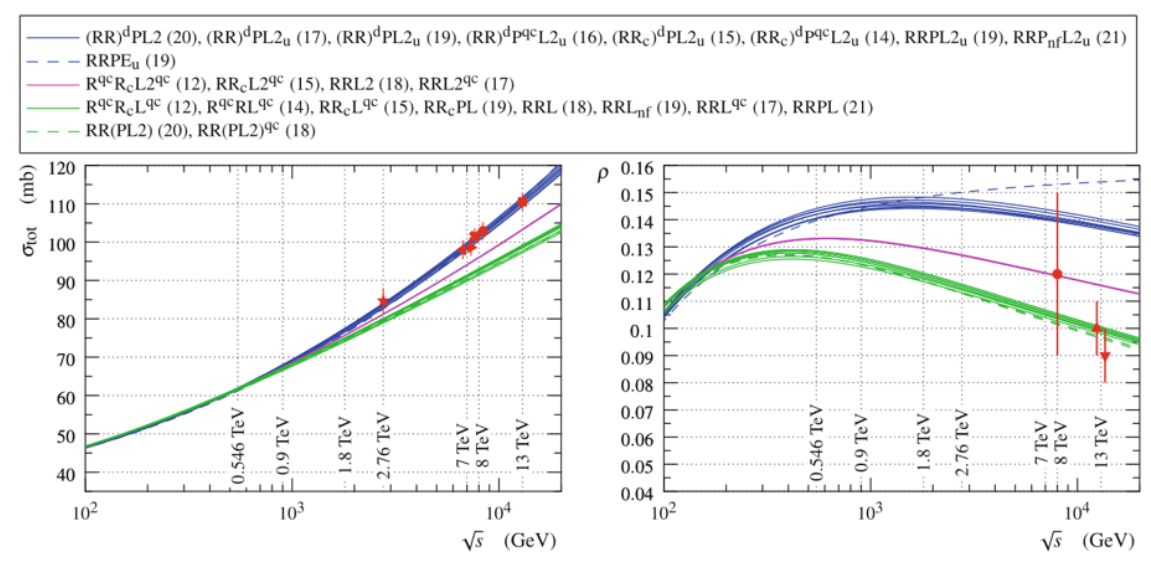

Figure 4: Predictions of COMPETE models for pp interactions. Each model is represented by one line (see legend). The red points represent the reference TOTEM measurements.

We combine the present analysis result with independent TOTEM odderon evidence based on the measurements of $\rho$ and $\sigma_{\text {tot }}$ for pp interaction at different $\sqrt{s}$. The $\rho$ and $\sigma_{\text {tot }}$ results are incompatible with models with Pomeron exchange only and provide independent evidence of odderon exchange effects with significance between $3.4 \sigma$ and $4.6 \sigma$ in completely different $|t|$ domains and TOTEM data sets, see Fig. 4 [13]. The full combination leads to total significances ranging from 5.2 to $5.7 \sigma$ for t-channel odderon exchange for all the models of Refs. [15] and [16].

\section{CONCLUSIONS}

In conclusion, we have compared the D0 pp elastic cross sections at $1.96 \mathrm{TeV}$ and the TOTEM pp cross sections extrapolated to $1.96 \mathrm{TeV}$ from measurements at $2.76,7,8$, and $13 \mathrm{TeV}$ using a data-driven method. The pp and $\mathrm{p} \overline{\mathrm{p}}$ cross sections differ with a significance of $3.4 \sigma$, and this standalone comparison provides evidence that a $t$-channel exchange of a colorless $\mathrm{C}$-odd gluonic compound, i.e., an odderon, is needed to describe elastic scattering at high energies [14]. When combined with the result of Ref. [13] the significance is in the range 5.2 to 5.7 $\sigma$ and thus constitutes the first experimental observation of the odderon. 


\section{References}

[1] V. M. Abazov et al. [TOTEM and D0], Phys. Rev. Lett. 127 (2021) no.6, 062003.

[2] V. M. Abazov et al. [D0], Nucl. Instrum. Meth. A 565 (2006), 463-537.

[3] V. M. Abazov et al. [D0], Phys. Rev. D 86 (2012), 012009.

[4] G. Antchev et al. [TOTEM], Int. J. Mod. Phys. A 28 (2013), 1330046.

[5] G. Antchev et al. [TOTEM], Eur. Phys. J. C 80 (2020) no.2, 91.

[6] G. Antchev et al. [TOTEM], EPL 95 (2011) no.4, 41001.

[7] G. Antchev et al. [TOTEM], Nucl. Phys. B 899 (2015), 527-546.

[8] G. Antchev et al. [TOTEM], Eur. Phys. J. C 79 (2019) no.10, 861.

[9] A. Breakstone et al., Phys. Rev. Lett. 54 (1985), 2180.

[10] E. Nagy, et al., Nucl. Phys. B 150 (1979), 221-267.

[11] M. Bozzo et al. [UA4], Phys. Lett. B 155 (1985), 197-202.

[12] D. Bernard et al. [UA4], Phys. Lett. B 171 (1986), 142-144.

[13] G. Antchev et al. [TOTEM], Eur. Phys. J. C 79 (2019) no.9, 785.

[14] E. Martynov and B. Nicolescu, Eur. Phys. J. C 79 (2019) no.6, 461.

[15] J. R. Cudell et al. [COMPETE], Phys. Rev. Lett. 89 (2002), 201801.

[16] V. A. Khoze, A. D. Martin and M. G. Ryskin, Phys. Lett. B 784 (2018), 192-198.

[17] A. L. Cholesky, Cours de l'Ecole Polytechnique, France (1910).

[18] R. A. Horn and C. R. Johnson, "Matrix analysis," Cambridge University Press (2012). 